This is an electronic reprint of the original article. This reprint may differ from the original in pagination and typographic detail.

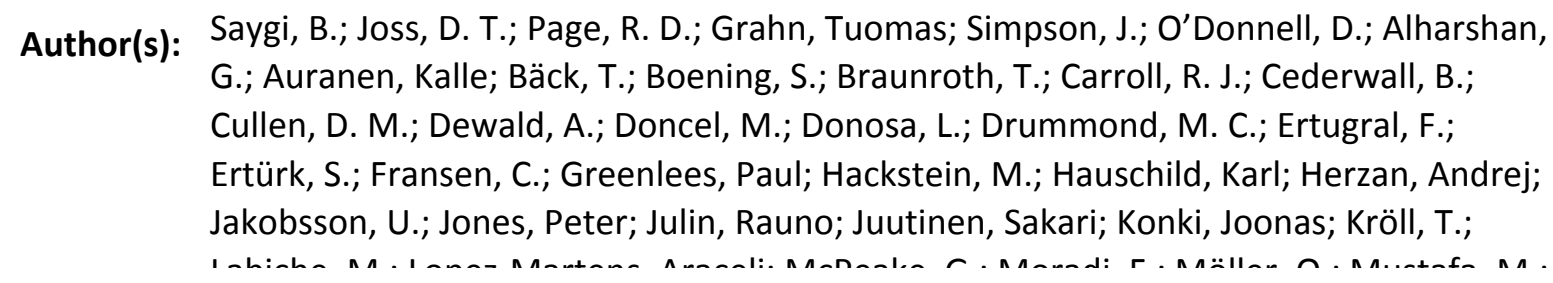

Title: $\quad$ Reduced transition probabilities along the yrast line in $166 \mathrm{~W}$

Year: $\quad 2017$

Version:

Please cite the original version:

Saygi, B., Joss, D. T., Page, R. D., Grahn, T., Simpson, J., O’Donnell, D., Alharshan, G., Auranen, K., Bäck, T., Boening, S., Braunroth, T., Carroll, R. J., Cederwall, B., Cullen, D. M., Dewald, A., Doncel, M., Donosa, L., Drummond, M. C., Ertugral, F., . . Uusitalo, J. (2017). Reduced transition probabilities along the yrast line in 166W. Physical Review C, 96(2), Article 021301(R). https://doi.org/10.1103/PhysRevC.96.021301

All material supplied via JYX is protected by copyright and other intellectual property rights, and duplication or sale of all or part of any of the repository collections is not permitted, except that material may be duplicated by you for your research use or educational purposes in electronic or print form. You must obtain permission for any other use. Electronic or print copies may not be offered, whether for sale or otherwise to anyone who is not an authorised user. 


\title{
Reduced transition probabilities along the yrast line in ${ }^{166} \mathrm{~W}$
}

B. Sayğ ${ }^{1,},{ }^{1,2}$ D. T. Joss, ${ }^{1}$ R. D. Page, ${ }^{1}$ T. Grahn, ${ }^{3}$ J. Simpson, ${ }^{4}$ D. O’Donnell,,${ }^{1,5}$ G. Alharshan, ${ }^{6}$ K. Auranen, ${ }^{3}$ T. Bäck, ${ }^{7}$ S. Boening, ${ }^{8}$ T. Braunroth,${ }^{9}$ R. J. Carroll, ${ }^{1}$ B. Cederwall, ${ }^{7}$ D. M. Cullen, ${ }^{6}$ A. Dewald,${ }^{9}$ M. Doncel,,${ }^{1}$ L. Donosa,${ }^{1}$

M. C. Drummond, ${ }^{1}$ F. Ertuğral,,${ }^{10}$ S. Ertürk, ${ }^{11}$ C. Fransen, ${ }^{9}$ P. T. Greenlees, ${ }^{3}$ M. Hackstein, ${ }^{9}$ K. Hauschild, ${ }^{3}$ A. Herzan, ${ }^{1}$ U. Jakobsson, ${ }^{7}$ P. M. Jones, ${ }^{3}$ R. Julin, ${ }^{3}$ S. Juutinen, ${ }^{3}$ J. Konki, ${ }^{3}$ T. Kröll,${ }^{8}$ M. Labiche, ${ }^{4}$ A. Lopez-Martens,${ }^{3}$ C. G. McPeake, ${ }^{1}$ F. Moradi, ${ }^{7}$ O. Möller, ${ }^{8}$ M. Mustafa, ${ }^{1}$ P. Nieminen, ${ }^{3}$ J. Pakarinen, ${ }^{3}$ J. Partanen,${ }^{3}$ P. Peura, ${ }^{3}$ M. Procter,${ }^{6}$ P. Rahkila, ${ }^{3}$ W. Rother, ${ }^{9}$ P. Ruotsalainen, ${ }^{3}$ M. Sandzelius, ${ }^{3}$ J. Sarén, ${ }^{3}$ C. Scholey,${ }^{3}$ J. Sorri, ${ }^{3}$ S. Stolze,${ }^{3}$ M. J. Taylor ${ }^{12}$ A. Thornthwaite, ${ }^{1}$ and J. Uusitalo ${ }^{3}$ ${ }^{1}$ Oliver Lodge Laboratory, Department of Physics, University of Liverpool, Liverpool L69 7ZE, United Kingdom

${ }^{2}$ Department of Physics, Faculty of Science, Ege University, Bornova, Izmir 35100, Turkey

${ }^{3}$ University of Jyvaskyla, Department of Physics, P.O. Box 35, FI-40014, University of Jyvaskyla, Finland

${ }^{4}$ STFC Daresbury Laboratory, Daresbury, Warrington WA4 4AD, United Kingdom

${ }^{5}$ School of Engineering and Computing, University of the West of Scotland, Paisley PA1 2BE, United Kingdom

${ }^{6}$ Department of Physics and Astronomy, University of Manchester, Manchester M13 9PL, United Kingdom

${ }^{7}$ Department of Physics, Royal Institute of Technology, SE-10691 Stockholm, Sweden

${ }^{8}$ Institut für Kernfysik, TU Darmstadt, Schlossgartenstrasse 9, D-64289 Darmstadt, Germany

${ }^{9}$ Institut für Kernphysik, Universität zu Köln, 50937 Köln, Germany

${ }^{10}$ Department of Physics, Faculty of Science and Arts, Sakarya University, 54100 Sakarya, Turkey

${ }^{11}$ Department of Physics, Faculty of Science and Arts, Omer Halisdemir University, 51200 Nigde, Turkey

${ }^{12}$ Division of Cancer Sciences, University of Manchester, Manchester M13 9PL, United Kingdom

(Received 7 July 2017; published 2 August 2017)

\begin{abstract}
Lifetimes of excited states in the yrast band of the neutron-deficient nuclide ${ }^{166} \mathrm{~W}$ have been measured utilizing the DPUNS plunger device at the target position of the JUROGAM II $\gamma$-ray spectrometer in conjunction with the RITU gas-filled separator and the GREAT focal-plane spectrometer. Excited states in ${ }^{166} \mathrm{~W}$ were populated in the ${ }^{92} \mathrm{Mo}\left({ }^{78} \mathrm{Kr}, 4 p\right)$ reaction at a bombarding energy of $380 \mathrm{MeV}$. The measurements reveal a low value for the ratio of reduced transitions probabilities for the lowest-lying transitions $B\left(E 2 ; 4^{+} \rightarrow 2^{+}\right) / B\left(E 2 ; 2^{+} \rightarrow 0^{+}\right)=$ $0.33(5)$, compared with the expected ratio for an axially deformed rotor $\left(B_{4 / 2}=1.43\right)$.
\end{abstract}

DOI: 10.1103/PhysRevC.96.021301

The advent of differential plunger lifetime measurements performed in conjunction with selective tagging techniques has allowed the nature of collectivity in highly neutron-deficient nuclei to be investigated [1-4]. Recent lifetime measurements of the lowest-lying yrast states in ${ }^{168}$ Os have revealed a ratio of reduced transition probabilities $B\left(E 2: 4^{+} \rightarrow 2^{+}\right) / B(E 2$ : $\left.2^{+} \rightarrow 0^{+}\right)=B_{4 / 2}=0.34(18)$, which is much lower than the collective limits expected for harmonic vibrators and axially deformed rotors that result in ratios of $B_{4 / 2}=2.00$ and 1.43, respectively [5,6]. A survey by Cakirli et al. revealed several nuclei with similarly low ratios [7] and further cases have been identified in the $A \sim 50$ [8], $A \sim 70$ [9], and $A \sim 110[10,11]$ mass regions. A global explanation of the anomaly has not yet been proposed. This paper discusses the yrast states of ${ }^{166} \mathrm{~W}$, which is a lighter isotone of ${ }^{168} \mathrm{Os}$ to investigate further this phenomenon.

Excited states in ${ }^{166} \mathrm{~W}$ were populated using the ${ }^{92} \mathrm{Mo}\left({ }^{78} \mathrm{Kr}, 4 p\right)$ reaction. A $380-\mathrm{MeV}^{78} \mathrm{Kr}^{15+}$ beam provided by the K130 cyclotron at the University of Jyväskylä Accelerator Laboratory bombarded a $0.6-\mathrm{mg} / \mathrm{cm}^{2}{ }^{92} \mathrm{Mo}$ target. A nominal beam intensity of 3 pnA was delivered to the target. The reaction provided an initial recoil velocity of $v / c=4.3 \%$. The $\gamma$ rays emitted by the recoiling nuclei were detected by the JUROGAM II $\gamma$-ray detector array consisting of 15 phase 1 or GASP-type detectors [12] and 24 Eurogam Clover germanium detectors [13]. All detectors were used in conjunction with escape-suppression shields.
The differential plunger for lifetime measurements of unbound nuclear states (DPUNS) [14] was installed at the JUROGAM II target position in order to measure excited-state lifetimes using the recoil distance Doppler shift (RDDS) technique [15]. A $1 \mathrm{mg} / \mathrm{cm}^{2}$ thick Mg degrader was employed to slow down evaporation residues to $v / c=3.3 \%$ yet allow reaction products to recoil into the RITU gas-filled separator [16-18] and be transported to its focal plane. The recoiling fusion-evaporation residues were implanted into the doublesided silicon strip detectors (DSSDs) of the GREAT spectrometer [19] located at the RITU focal plane. The energy loss of the recoils, measured in the multiwire proportional counter, and time of flight (in conjunction with the DSSDs) were used to discriminate fusion-evaporation residues from scattered beam. The GREAT triggerless data acquisition system [20] was utilized to collect data time stamped to a precision of $10 \mathrm{~ns} . \gamma$-ray spectra in delayed coincidence with the implanted recoils were sorted with the GRAIN data analysis package [21].

Recoil-correlated $\gamma$-ray coincidences were recorded at nine target-to-degrader distances of the DPUNS device ranging from 5 to $8000 \mu \mathrm{m}$. This range was chosen to span the region of sensitivity where the relative intensities of the fully shifted and degraded components of the depopulating transitions for the low-lying yrast states in ${ }^{166} \mathrm{~W}$ varied. Examples of typical spectra are shown in Fig. 1 while a partial level scheme for the yrast band of ${ }^{166} \mathrm{~W}$ is shown in Fig. 2. 


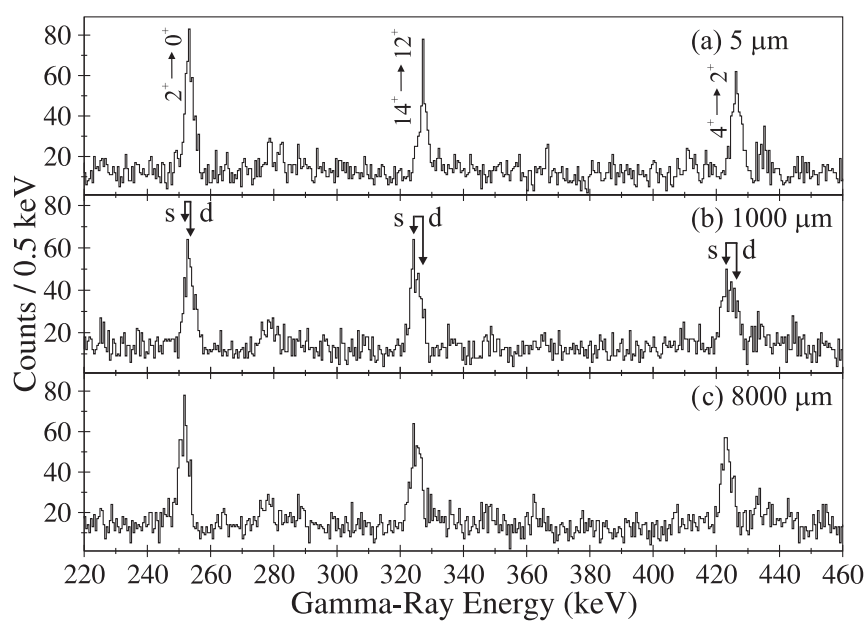

FIG. 1. $\gamma$-ray coincidences correlated with implanted nuclear recoils detected in the DSSDs of the GREAT spectrometer. Spectra for three different target-to-degrader distances of (a) 5, (b) 1000, and (c) $8000 \mu \mathrm{m}$ with the ten JUROGAM II Ge detectors at $133^{\circ}$. Each spectrum is in coincidence with the $480 \mathrm{keV}\left(12^{+} \rightarrow 10^{+}\right)$transition, which was chosen to eliminate the contributions from energy doublets in the side bands. The fully Doppler-shifted (s) and degraded (d) components of the 252-, 326-, and 424-keV $\gamma$-ray transitions are labeled.

Sufficient $\gamma$-ray coincidences were collected with JUROGAM II between the detection angles at $158^{\circ}$ (five detectors) and $133^{\circ}$ (ten detectors) with all other detectors to allow the measurements of some yrast states using the differential decay curve method (DDCM) [15]. The NAPATAU software was used to analyze $\gamma$-ray intensities. In the DDCM, the mean lifetimes are obtained from the relative intensity variation with target-to-degrader distance of the fully Dopplershifted and degraded components of the $\gamma$-ray transitions feeding and depopulating the level of interest through the equation

$$
\tau=\frac{Q_{\text {depop }}^{d}(x)-Q_{\text {feed }}^{d}(x)}{v \frac{d}{d x}\left[Q_{\text {depop }}^{s}(x)\right]},
$$

where $Q_{j}^{i}(x)=I_{j}^{i} /\left(I_{j}^{s}+I_{j}^{d}\right)$ and $I_{j}^{i}(x)$ are the $\gamma$-ray intensities for the shifted $(i=s)$ and degraded $(i=d)$ components measured at the target-to-degrader distance $x$ for the depopulating ( $j=$ depop) and feeding $(j=$ feed) transitions, respectively. Therefore, the $\gamma$-ray intensities $I$ recorded with different distances $x$ are normalized by the sum of their fully shifted and degraded components. The final lifetime is an error-weighted average of individual lifetimes [Eq. (1)] obtained at the different target-to-degrader distances within the region of sensitivity where the derivative of the decay curve is greater than zero. The lifetime determination analysis for the $2^{+}$and $4^{+}$states is shown in Fig. 3 .

In order to extract the lifetimes of the $2^{+}, 4^{+}$, and $6^{+}$states, respectively, $\gamma$-ray coincidences were demanded between the full line shape of the $480-\mathrm{keV}\left(12^{+} \rightarrow 10^{+}\right)$and $686-\mathrm{keV}\left(10^{+} \rightarrow 8^{+}\right)$direct feeding transitions to eliminate the influence of energy doublets and unobserved feeding transitions on the lifetimes under investigation. Coincidences

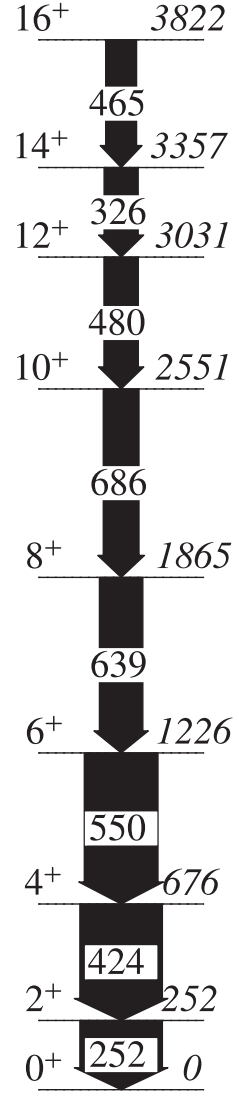

(a)
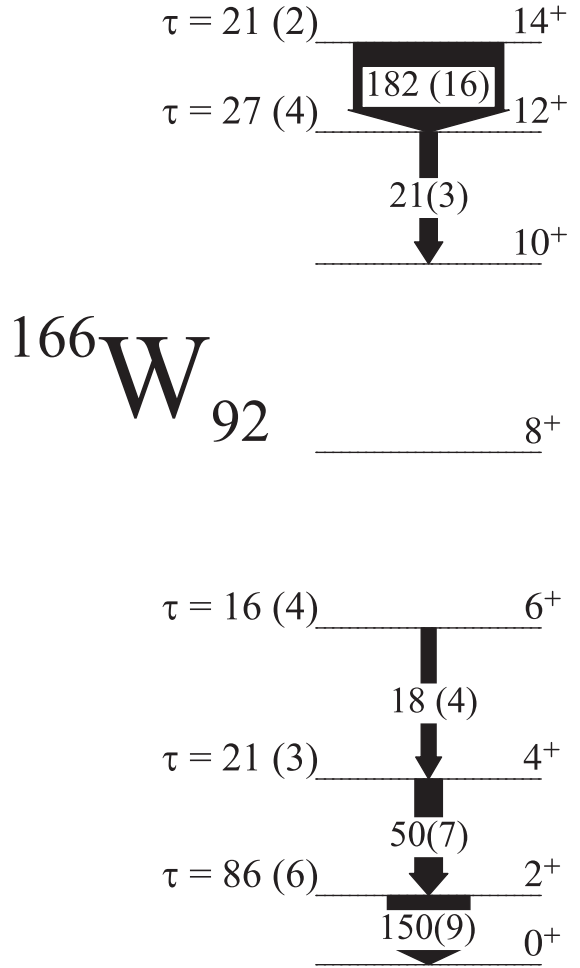

(b)
FIG. 2. (a) Partial level scheme showing levels and transitions in the yrast band of ${ }^{166} \mathrm{~W}$. The width of the arrows is proportional to the relative intensities of the $\gamma$-ray transitions. The levels are labeled by their spins, parities, and excitation energies. All energies are stated in $\mathrm{keV}$. (b) Partial level scheme for the yrast band in ${ }^{166} \mathrm{~W}$ with the levels labeled by their spins, parities, and mean lifetimes in picoseconds. The width of the arrows is proportional to the reduced transition probabilities of the $\gamma$-ray transitions with values given in Weisskopf units $(\mathrm{Wu})$.

were demanded with the whole JUROGAM II spectrometer, and the lower-lying depopulating transitions recorded in the JUROGAM II detectors at $133^{\circ}$ or $158^{\circ}$. The indirect $\gamma$-ray feeding intensities were measured by demanding coincidences below the states of interest. This method has been used previously in Ref. [22].

In order to extract the lifetimes of the $12^{+}$and $14^{+}$states, the spectra for the feeding and depopulating transitions were obtained by demanding coincidences with the full $\gamma$-ray lineshape of transitions directly depopulating and feeding the levels of interest, respectively $[22,23]$. The measured mean lifetimes $\tau$ and the deduced $B(E 2)$ reduced transition probabilities are listed Table I and displayed in Fig. 2(b). At high spin, the $B\left(E 2 ; 12^{+} \rightarrow 10^{+}\right)=21(3) \mathrm{Wu}$ is measured to be much lower than the preceding transition $\left[B\left(E 2 ; 14^{+} \rightarrow 12^{+}\right)\right.$ $=182(16) \mathrm{Wu}]$. This feature is similar to the measurement of a long $12^{+}$state lifetime in ${ }^{168} \mathrm{~W}$ [24]. Dracoulis et al. attributed this to a weak interaction between the ground and $i_{13 / 2}^{2}$ aligned 


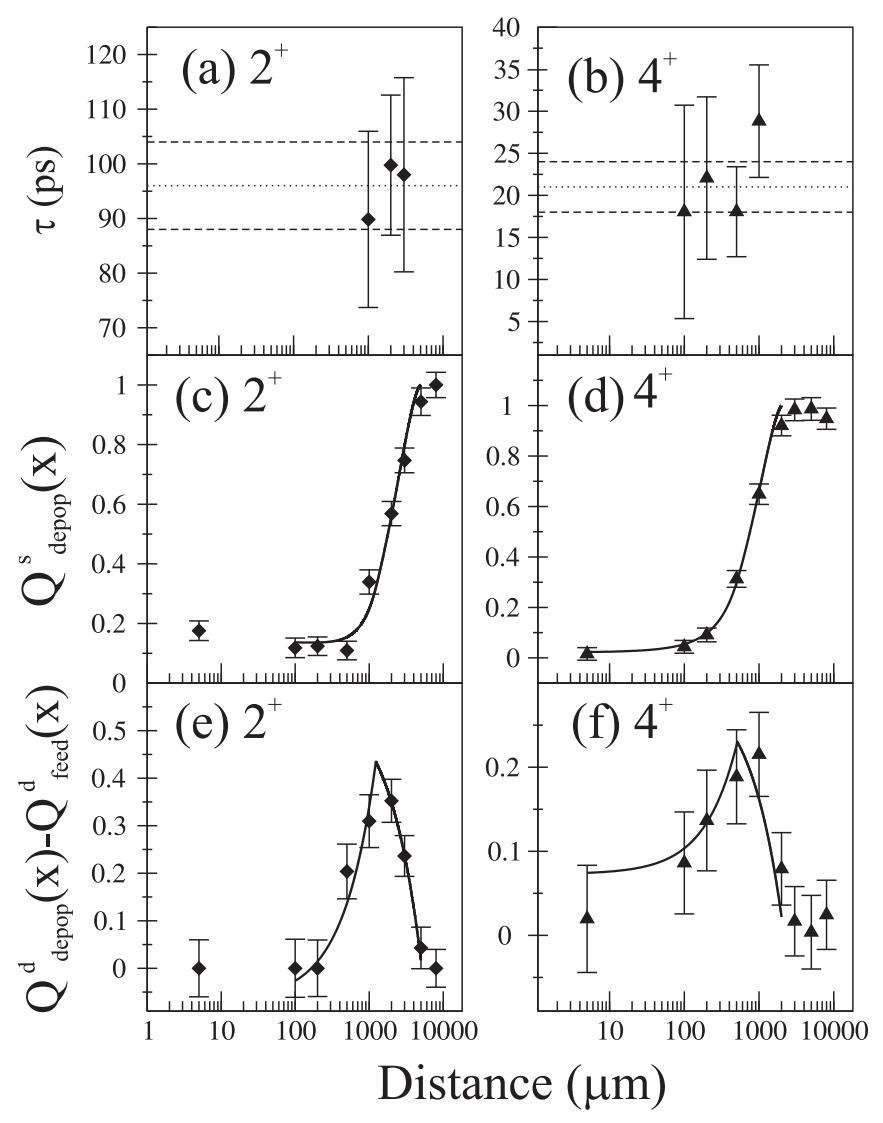

FIG. 3. Lifetime determination using the differential decay curve method (DDCM). The individual mean lifetimes and error bars are represented as the solid and dashed horizontal lines, respectively for (a) the $2^{+}$state and (b) the $4^{+}$state in ${ }^{166} \mathrm{~W}$. The decay curves extracted from coincidence spectra gated above the (c) $2^{+}$state and (d) $4^{+}$state. The line drawn through the experimental points is the fit to the decay curve. All spectra are collected from the ten detectors at $133^{\circ}$. (e) The numerator of Eq. (1) and the derivative of the decay curve for the $2^{+}$state and (f) the $4^{+}$state.

band configurations in the backbending region, resulting in a low degree of level mixing [24].

At low spin, the evolution of collective behavior is reflected in the excitation energies of the low-lying excited states. Figure 4(a) shows the variation in the ratio of the excitation energies of the yrast $4^{+}$to $2^{+}$states in the $\mathrm{W}$ isotopes as a function of the neutron number. The systematic trends show ratios consistent with the transition from collective vibrations at ${ }^{160} \mathrm{~W}_{86}$ [29], through $\gamma$-soft rotors $\left({ }^{166} \mathrm{~W}_{92}\right)$ [30]

TABLE I. Lifetimes and reduced transition probabilities of the yrast states in ${ }^{166} \mathrm{~W}$.

\begin{tabular}{lcccc}
\hline \hline$E_{\gamma}[\mathrm{keV}]$ & $I_{i}^{\pi} \rightarrow I_{f}^{\pi}$ & $\tau(\mathrm{ps})$ & $B(E 2)\left[e^{2} b^{2}\right] \downarrow$ & $B(E 2)[$ W.u. $]$ \\
\hline 252 & $2^{+} \rightarrow 0^{+}$ & $86(6)$ & $0.81(5)$ & $150(9)$ \\
424 & $4^{+} \rightarrow 2^{+}$ & $21(3)$ & $0.27(3)$ & $50(7)$ \\
550 & $6^{+} \rightarrow 4^{+}$ & $16(4)$ & $0.09(2)$ & $18(4)$ \\
480 & $12^{+} \rightarrow 10^{+}$ & $27(4)$ & $0.11(1)$ & $21(3)$ \\
326 & $14^{+} \rightarrow 12^{+}$ & $21(2)$ & $0.98(8)$ & $182(16)$ \\
\hline \hline
\end{tabular}

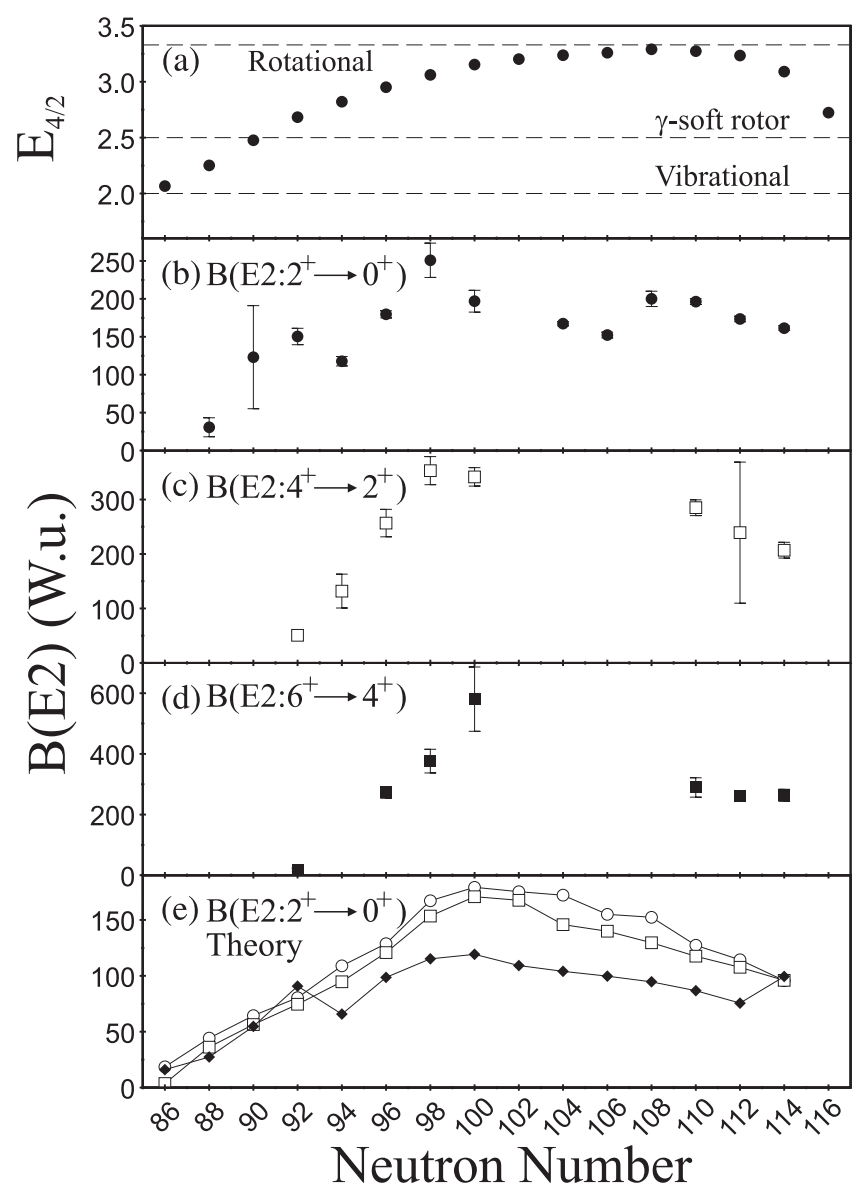

FIG. 4. (a) Ratios of the $4^{+}$and $2^{+}$state excitation energies $\left(E_{(4 / 2)}\right)$ for the even-mass W isotopes. Measured reduced transition probabilities $B(E 2)$ for the $\mathrm{W}$ isotopes for (b) the $2^{+} \rightarrow 0^{+}$ transitions, (c) the $4^{+} \rightarrow 2^{+}$transitions, and (d) the $6^{+} \rightarrow 4^{+}$ transitions. (e) Theoretical reduced transition probabilities $B(E 2)$ for the $2^{+} \rightarrow 0^{+}$transitions in the even-mass W isotopes. Model predictions using the finite-range droplet model (open diamonds), Woods-Saxon model (filled diamonds), and dynamical microscopic model (open circles) are displayed. The values for ${ }^{166} \mathrm{~W}$ correspond to neutron number $N=92$. Data for the heavier isotopes were obtained from Refs. [4,25-28]. All $B(E 2)$ values are given in Weisskopf units (Wu).

to well-deformed rotors near the neutron midshell $\left({ }^{178} \mathrm{~W}_{104}\right)$ [31]. As the neutron number increases further and the valence space reduces toward $N=126$, the excitation energy ratio decreases toward values consistent with $\gamma$-soft nuclei near ${ }^{190} \mathrm{~W}_{116}$ [32].

The measurement of reduced transition probabilities, $B(E 2)$ values, can provide more detailed insights into the development of collectivity. Figures 4(b)-4(d) shows the variation of the $B(E 2)$ values for low-lying transitions as a function of the neutron number. The $B\left(E 2 ; 2^{+} \rightarrow 0^{+}\right)$value reaches its maximum value of $\sim 200$ W.u at $N \sim 96$ as the $E_{4 / 2}$ ratio approaches the rotational limit $\left(E_{4 / 2}=3.33\right)$. The $B\left(E 2 ; 4^{+} \rightarrow 2^{+}\right)$and $B\left(E 2 ; 6^{+} \rightarrow 4^{+}\right)$values are very low at $N=92$ and increase steadily as the number of valence neutrons approaches the neutron midshell $(N=104)$. 
Theoretical model predictions for $B\left(E 2 ; 2^{+} \rightarrow 0^{+}\right)$values have been compiled by Raman et al. for the $2 \leqslant Z \leqslant 100$ nuclei [33]. Figure 4(e) shows a comparison between the predictions by the finite-range droplet model, Woods-Saxon model, and dynamical microscopic model for the $B\left(E 2 ; 2^{+} \rightarrow\right.$ $0^{+}$) values in the tungsten isotopes. Note that in order to allow comparisons with our measured values, the $B\left(E 2 ; 2^{+} \rightarrow\right.$ $\left.0^{+}\right) \uparrow$ measured and theoretical values of Ref. [33] have been converted into $B\left(E 2 ; 2^{+} \rightarrow 0^{+}\right) \downarrow$ values using the expression

$$
B\left(T \lambda: I_{2} \rightarrow I_{1}\right)=\frac{2 I_{1}+1}{2 I_{2}+1} B\left(T \lambda: I_{1} \rightarrow I_{2}\right) .
$$

In general, all three models predict qualitatively the behavior of experimental measurements for the $N \geqslant 86$ isotopes. While the finite-range droplet model and Woods-Saxon model calculations fail to reproduce the measured experimental $B\left(E 2 ; 2^{+} \rightarrow 0^{+}\right)$dip at $N=94$ the dynamical microscopic model appears to reproduce this feature for ${ }^{168} \mathrm{~W}$. The dynamical microscopic model employs generator coordinate method techniques, which may be better suited for describing transitional nuclei through the microscopic determination of the collective dynamics $[34,35]$.

The experimental ratios of reduced transition probabilities $B_{4 / 2}, B_{6 / 2}$, and $B_{6 / 4}$ in ${ }^{166} \mathrm{~W}$ are compared with the heavier $\mathrm{W}$ isotopes and the theoretical collective rotational limits [36] in Fig. 5. The $B\left(E 2 ; 2^{+} \rightarrow 0^{+}\right)$measurements yield higher values than both the $B\left(E 2 ; 4^{+} \rightarrow 2^{+}\right)$and $B\left(E 2 ; 6^{+} \rightarrow 4^{+}\right)$ measurements at $N=92$, which results in an anomalously low ratios of reduced transition probabilities at $B_{4 / 2}=0.33(5)$ and $B_{6 / 2}=0.12(3)$, respectively. The measured $B_{6 / 4}=0.36(11)$ ratio is much lower than those extracted for the heavier isotopes, see Fig. 5(c), which lie close to the theoretical limit of $B_{6 / 4}=1.1$, indicating that the irregularity is not solely related to the structure of the yrast $2^{+}$state.

The $B_{4 / 2}$ ratio in ${ }^{166} \mathrm{~W}$ shows a remarkable similarity to that measured recently for the heavier $N=92$ isotone ${ }^{168} \mathrm{Os}$ $\left[B_{4 / 2}=0.34(18)\right]$ [3]. The origin of the anomaly in both cases remains an enigma, but some typical reasons for such features can be discounted. For example, the lack of proximity to a closed shell precludes an inversion due to a generalized seniority scheme [37]. Shape coexistence effects are also unlikely to provide an explanation. Shape coexistence has been observed in ${ }^{172}$ Os [38,39], resulting in perturbed low-lying yrast states but an anomalous $B_{4 / 2}$ ratio has not been measured for this isotope [40]. Moreover, band-mixing calculations for ${ }^{168}$ Os indicate that a similar perturbation for the unexpected ratios is not likely [41]. The only remaining candidate for the feature in ${ }^{168} \mathrm{Os}$ is mixing between states in the ground-state band and low-lying states in a nonyrast positive-parity band [3]. However, no analogous band has been observed in ${ }^{166} \mathrm{~W}$, which is consistent with the systematic trends established for the $\mathrm{W}$ isotopes [42].

It has been suggested that anomalous $B_{4 / 2}$ ratios in the $\gamma$-soft nuclei such as ${ }^{168} \mathrm{Os}$, and therefore by extension ${ }^{166} \mathrm{~W}$, may arise from mixing due to dynamical shape fluctuations [3]. While beyond the scope of this work, a detailed theoretical study of such effects might reveal the underlying physical

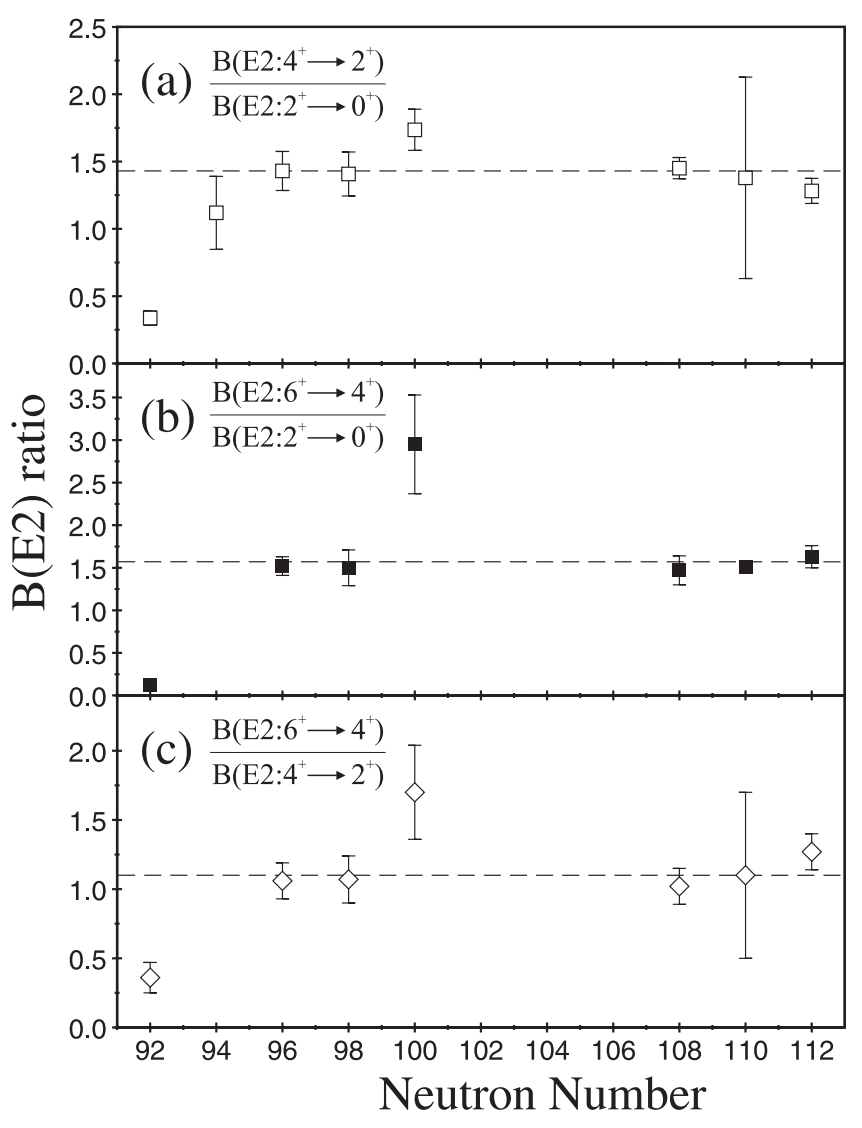

FIG. 5. Ratios of reduced transition probabilities measured for the even-mass W isotopes (a) $B\left(E 2: 4^{+} \rightarrow 2^{+}\right) / B\left(E 2: 2^{+} \rightarrow 0^{+}\right)$, (b) $B\left(E 2: 6^{+} \rightarrow 4^{+}\right) / B\left(E 2: 2^{+} \rightarrow 0^{+}\right)$, and (c) $B\left(E 2: 6^{+} \rightarrow 4^{+}\right) /$ $B\left(E 2: 4^{+} \rightarrow 2^{+}\right)$. In each case, the theoretical ratio for a collective rotor is indicated by a dashed line [36].

basis for the unusual nature of the anomalous ratios of reduced transition probabilities in these $\gamma$-soft transitional nuclei.

In summary, the lifetimes of $2^{+}, 4^{+}, 6^{+}, 12^{+}$, and $14^{+}$ states in ${ }^{166} \mathrm{~W}$ have been measured for the first time using the recoil-distance Doppler shift method. Reduced transition probabilities extracted from these lifetimes of the low-lying states in the ground-state band have revealed abnormal $B_{4 / 2}$ and $B_{6 / 2}$ ratios with values of $0.33(5)$ and $0.12(3)$, respectively, which are lower than the values expected from collective rotational models $\left(B_{4 / 2}=1.43\right.$ and $\left.B_{4 / 2}=1.57\right)$. The structural origin of the anomaly remains enigmatic and further work is required to investigate whether the expected $\gamma$ softness of ${ }^{166} \mathrm{~W}$ might contribute to this puzzling observation.

This work has been supported through EURONS (European Commission Contract No. RII3-CT-2004-506065), the Academy of Finland under the Finnish Centre of Excellence Programme 2006-2011 (Nuclear and Accelerator Based Physics Contract No. 213503), and the UK Science and Technology Facilities Council. The UK/France (STFC/IN2P3) Loan Pool and GAMMAPOOL network are acknowledged for the EUROGAM detectors of JUROGAM II. T.G. acknowledge the support of the Academy of Finland (Contract No. 131665). 
[1] M. G. Procter et al., Phys. Lett. B 725, 79 (2013).

[2] D. Hodge et al., Phys. Rev. C 94, 034321 (2016).

[3] T. Grahn et al., Phys. Rev. C 94, 044327 (2016).

[4] M. Doncel et al., Phys. Rev. C 95, 044321 (2017).

[5] A. Bohr and B. R. Mottelson, Nuclear Structure Volume 2: Nuclear Deformation (W. A. Benjamin, New York, 1975).

[6] A. Arima and F. Iachello, Ann. Phys. 123, 468 (1979).

[7] R. B. Cakirli, R. F. Casten, J. Jolie, and N. Warr, Phys. Rev. C 70, 047302 (2004).

[8] D. Hertz-Kintish, L. Zamick, and S. J. Q. Robinson, Phys. Rev. C 90, 034307 (2014).

[9] C. Louchart et al., Phys. Rev. C 87, 054302 (2013).

[10] G. de Angelis et al., Phys. Lett. B 535, 93 (2002).

[11] O. Möller et al., Phys. Rev. C 71, 064324 (2005).

[12] C. W. Beausang et al., Nucl. Instrum. Methods Phys. Res. A 313, 37 (1992).

[13] G. Duchene et al., Nucl. Instrum. Methods Phys. Res. A 432, 90 (1999).

[14] M. J. Taylor et al., Nucl. Instrum. Methods Phys. Res. A 707, 143 (2013).

[15] A. Dewald, S. Harissopulos, and P. von Brentano, Z. Phys. A 334, 163 (1989).

[16] M. Leino et al., Nucl. Instrum. Methods Phys. Res. B 99, 653 (1995).

[17] J. Uusitalo et al., Nucl. Instrum. Methods Phys. Res. B 204, 638 (2003).

[18] J. Saren, J. Uusitalo, M. Leino, and J. Sorri, Nucl. Instrum. Methods Phys. Res. A 654, 508 (2011).

[19] R. D. Page et al., Nucl. Instrum. Methods Phys. Res. B 204, 634 (2003).

[20] I. H. Lazarus et al., IEEE Trans. Nucl. Sci. 48, 567 (2001).

[21] P. Rahkila, Nucl. Instrum. Methods Phys. Res. A 595, 637 (2008).

[22] A. Dewald et al., Phys. Rev. C 68, 034314 (2003).

[23] T. Grahn et al., Phys. Rev. C 80, 014324 (2009).

[24] G. D. Dracoulis, G. D. Sprouse, O. C. Kistner, and M. H. Rafailovich, Phys. Rev. C 29, 1576 (1984).
[25] W. V. Werner et al., J. Phys. G 312, 092062 (2011).

[26] M. Rudigier, J.-M. Regis, J. Jolie, K. Zell, and C. Fransen, Nucl. Phys. A 847, 89 (2010).

[27] W. M. Regis et al., Nucl. Instrum. Methods. Phys. Res. A 606, 466 (2009).

[28] Data extracted using the NNDC On-Line Data Service from the ENSDF database, file revised as of 22.2.2010. M. R. Bhat, Evaluated Nuclear Structure Data File (ENSDF), in Nuclear Data for Science and Technology, edited by S. M. Qaim (Springer-Verlag, Berlin, 1992), p. 817.

[29] A. Keenan et al., Phys. Rev. C 63, 064309 (2001).

[30] J. Simpson, F. Hanna, M. A Riley, A. Alderson, M. A. Bentley, A. M. Bruce, D. M. Cullen, P. Fallon, and L. Walker, J. Phys. G 18, 1207 (1992).

[31] M. J. Canty, N. E. Davison, D. A. Dohan, and P. Yuen, Nucl. Phys. A 265, 1 (1976).

[32] Z. Podolyak et al., Phys. Lett. B 491, 225 (2000).

[33] S. Raman, C. W. Nestor, and P. Tikkanen, At. Data Nucl. Data Tables 78, 1 (2001).

[34] P. G. Reinhard and K. Goeke, Rep. Prog. Phys. 50, 1 (1987).

[35] N. Onishi and S. Yoshida, Nucl. Phys. 80, 367 (1966).

[36] D. J. Rowe and J. L. Wood, Fundamentals of Nuclear Models: Foundational Models (World Scientific, Singapore, 2009), Vol. 1.

[37] A. de Shalit and I. Talmi, Nuclear Shell Theory (Academic Press, New York, 1963).

[38] J. L. Durell, G. D. Dracoulis, C. Fahlander, and A. P. Byrne, Phys. Lett. B 115, 367 (1982).

[39] G. D. Dracoulis, R. A. Bark, A. E. Stuchbery, A. P. Byrne, A. M. Baxter, and F. Reiss, Nucl. Phys. A 115, 486 (1982).

[40] A. Virtanen, N. R. Johnson, F. K. McGowan, I. Y. Lee, C. Baktash, M. A. Riley, J. C. Wells, and J. Dudek, Nucl. Phys. A 591, 145 (1995).

[41] D. T. Joss et al., Nucl. Phys. A 689, 631 (2001).

[42] T. Kibedi, G. D. Dracoulis, A. P. Byrne, and P. M. Davidson, Nucl. Phys. A 688, 669 (2001). 